\title{
Ring current decay time model during geomagnetic storms: a simple analytical approach
}

\author{
R. Monreal MacMahon and C. Llop-Romero \\ University of Magallanes, CEQUA, Space Physics Lab, Casilla 113-D, Punta Arenas, Chile
}

Received: 24 September 2007 - Revised: 12 June 2008 - Accepted: 17 July 2008 - Published: 3 September 2008

\begin{abstract}
The ring current growth and decay, characterized by the $D_{s t}$ index, has been studied for thirty years using the Burton et al. (1975) equation. The original formula is based on the restriction of the DPS (Dessler, Parker, and Schoppke) theorem and assuming a constant decay time of particles. The decay time scale is important because the energy injection rate cannot be determined it without the knowledge of this parameter. In a previous work, instead of using a constant value, we introduced the decay time of particles in the energy rate balance equation as a continuous function of the absolute value of the pressure corrected $D_{s t}$ index to avoid the reported discontinuities determining it. Here, based on the DPS restriction, we extend our previous empirical work to obtain analytically the proposed continuous function considering losses due to a global resistive force as a product of viscous-like, and other related dissipation processes. We test our model predicting $D_{s t}$ for a couple of specific storm events and also comparing our results with forecasts of a good reference model appeared in the literature.
\end{abstract}

Keywords. Magnetospheric physics (Current systems; Solar wind-magnetosphere interactions; Storms and substorms)

\section{Introduction}

As a result of the solar wind-magnetosphere coupling there is a dynamo transferring energy into the inner magnetosphere which is partially injected and dissipated in the ring current belt. This ring is a toroidal current that flows in the magnetosphere of the Earth between 2 and 10 Earth radii $\left(R_{E}\right)$ (e.g. Gonzalez et al., 1994) and is characterized by the $D_{s t}$ index in such a way that an enhancement in the ring current is followed by a depression of the $D_{s t}$ index.

Correspondence to: R. Monreal MacMahon (rmonreal@ona.fi.umag.cl)
The energy balance of the current is carried by geomagnetically trapped energetic ions and electrons with energies between 10 and $200 \mathrm{keV}$ (e.g. Frank, 1967; Williams, 1981).

The actual mechanism that adds particles to the ring current is not completely understood (Daglis et al., 1999). It has been widely accepted that the access and energization of charged particles occurs during the main phase of a magnetic storm which lasts between approximately 3 and $12 \mathrm{~h}$ and is determined by the polar cap potential and the plasma sheet number density, that is, the inward transport of plasma sheet particles is driven by the enhanced convection electric field (e.g. Williams, 1981). However, Sun and Akasofu (2000) have shown that the formation of the ring current belt during geomagnetic storms is not just a result of an enhanced convection which is directly driven by the solar wind and that substorm processes are crucial in populating the ring current by $\mathrm{O}^{+}$ions.

The loss mechanisms that cause the recovery phase of the storm are fairly well documented. The recovery phase is due to largely collisional processes that causes the ring current to subsequently decay to its original quiet level on a typical time scale of approximately 2 and 3 days. Ebihara and Ejiri (2000) have reported that ions are lost by charge-exchange with neutral hydrogen and convection outflow to the dayside magnetopause (azimuthally located at $L=10$ ), neglecting the Coulomb collision loss with thermal plasma, the wave-particle interaction, and the loss cone loss processes. They show that the charge-exchange contributes significantly and $D_{s t}^{*}$ hardly recovers without this process during the late recovery phases.

It is well known that the temporal evolution of the ring current energy can be determined by the energy rate balance equation

$\frac{d K_{R}}{d t}=U_{R}-U_{L}$

Published by Copernicus Publications on behalf of the European Geosciences Union. 
where $U_{R}$ is the injection rate of energy and $U_{L}=\left(K_{R} / \tau\right)$ is due to the rate of energy loss. $K_{R}$ and $\tau$ are the kinetic energy of particles in the ring current and the associated decay time, respectively.

On the other hand, Dessler and Parker (1959) and Sckopke (1966) have shown that the ground magnetic field perturbation $\Delta B$ is related with the total energy $E$ of particles by the DPS expression

$$
\frac{\Delta B(0)}{B_{0}}=-\frac{2}{3} \frac{E}{E_{m}}
$$

where $E_{m}=\frac{4 \pi}{3} R_{E}^{3} B_{0}^{2} / \mu_{0}\left(=8 \times 10^{24} \mathrm{ergs}\right)$ is the total energy of the outer magnetic field ( $\mu_{0}$ is the vacuum magnetic susceptibility), $B_{0}$ is the horizontal geomagnetic field (0.3 gauss), and $\Delta B_{z}(0)$ is the ring current magnetic field in the Earth's centre and can be approximated by the absolute value of the $D_{s t}$ index. This approximation is valid when (Akasofu and Chapman, 1972), i) the ring current is symmetric in relation with the dipole axis, ii) the non-linear distortion of the geomagnetic field due to the ring current is not important. So, even though $D_{s t}$ also includes the nonsymmetric partial ring current, we assume that when the $D_{s t}$ index has a negative value it gives the field of the symmetric ring current. However, the magnetic field produced by the ring current is given by the pressure corrected $D_{s t}$ index, that is (e.g. Gonzalez et al., 1989)

$D_{s t}^{*}=D_{s t}-b \sqrt{p}+c$,

where $p=\rho v^{2}$ is the disturbed ram pressure of the solar wind, ( $\rho$ and $v$ are the solar wind density and velocity, respectively), $b=15.8 \mathrm{nT} / \sqrt{\mathrm{nPa}}$ is a constant value which represent a typical factor of proportionality for intense storms (Gonzalez et al., 1989) and $c=20 \mathrm{nT}$ gives the quiet-day contribution to $D_{s t}^{*}$. More recently, O'Brien and McPherron (2000a) obtained correction values of $b=7.26 \mathrm{nT} / \sqrt{\mathrm{nPa}}$ and $c=11 \mathrm{nT}$. As noted above, the expression for $D_{s t}^{*}$ involves only ram pressure correction. An additional correction to $D_{s t}^{*}$ due to induced current in the solid Earth (e.g. Dessler and Parker, 1959; Langel and Estes, 1983; Stern, 1984) has been usually neglected in the literature and it was not taken into account here. Such a correction typically reduces the value of $D_{s t}^{*}$ by a factor of $1 / 3$ (e.g. Akasofu and Chapman, 1972; Langel and Estes, 1985; Gonzalez et al., 1994), so the values obtained from Eq. (3) tend to overestimate $U_{R}$ if we consider the DPS restriction.

Usually, the study of the ring current dynamics has been focused on an analysis of the $D_{s t}$ index. Additionally to be a measure of the magnetic field of the ring current flowing in the magnetosphere, the absolute value $D$ of $D_{s t}^{*}$ is also a measure of the kinetic energy $K_{R}$ of the particles that make up the toroidal current. Then, from the DPS equation (Eq. 2) follows $D / B_{0}=\frac{2}{3} K_{R} / E_{m}$ and the usual energy balance equa- tion (Eq. 1) can be written as an evolution equation for $D$ (Burton et al., 1975)

$\frac{d D}{d t}+\frac{D}{\tau}=-Q$,

where $Q$ is the energy related input function, which is usually considered as directly proportional to the interplanetary electric field $v B_{S}$ (e.g. O'Brien and McPhherron, 2000), that is

$Q=-4.4\left(v B_{S}-E_{C}\right)$,

when $v B_{S}>E_{C}=0.49 \mathrm{mV} / \mathrm{m}$, otherwise $Q=0$.

The parameter $\tau$ in Eq. (4) corresponds to the decay time of particles which has been observed to be much shorter during the early recovery phase of very intense storms than during the posterior recovery.

The behavior of tau with the recovery phase proceeding has been explained in connection with the tail current contribution to $D_{s t}$ (e.g. Maltsev, 2004, and references therein) considering that the cross-tail current decays more rapidly than the ring current because it is more directly related to the changing convection electric fields imposed by solar wind interactions. Another previous explanation is connected to the contribution of $\mathrm{O}+$ ions of ionospheric origin to the storm time particle content (e.g. Daglis et al., 2003). That is, the ring current decay time should depends not only to changing convection electric fields imposed by solar wind interactions but to internal dynamics of the inner magnetosphere system.

We use the simple formula of Burton et al., which have been successfully used for more than thirty years, but considering the decay time parameter as a continuous function of the absolute value of the $D_{s t}^{*}$ index (Monreal MacMahon and Gonzalez, 1997). Here, based on the DPS restriction, which relates $D_{s t}$ to the internal dynamics, we extend our previous empirical work to obtain analytically the proposed continuous function making some assumptions in the system condition. As explained by Zhang et al. (2007), "under the enhanced convection electric fields and geomagnetic fields, a mixture of the fresh ionospheric and solar wind plasmas, together with the magnetospheric plasmas, is energized and undergoes large-scale drift in the global magnetosphere. Some of the particles can be transported inward enough to form the storm-time ring current". Our simple model is based on a global large-scale current flux which is associated to different flux sources whether of ionospheric origin or magnetotail origin driven by the solar wind, involving both previous explanations.

It is interesting to note that for a very complex system we can use a simple model which can be obtained through reliable physical assumptions reduced to a global resistive force which explains partially the decay time of particles and makes possible to do a good $1 \mathrm{~h}-D_{s t}$ prediction. In Sect. 2 we review different decay time models. In Sect. 3 we derive the functional dependence of the decay time parameter. The validation of the model is done in Sect. 4. 


\section{The decay time parameter}

Previous works (e.g. Prigancová and Feldstein, 1992) have shown that a reconstitution of the ring current energization process require an adequate estimate of the decay time of particles in the ring current. As was stated by Akasofu (1986), a better knowledge and estimate of $\tau$ will give us a greater insight of the energy input - output in the magnetosphere. As the energy injection rate $U_{R}$ and consequently the total energy rate $U_{T}$ can not be determined without using the decay time scale, a detailed knowledge of this property is of great interest.

Diverse works have been done on $\tau$ estimate. Some of them have proposed constant values of the decay time for all possible $D_{s t}$ values (e.g. Burton et al., 1975; Perrault and Akasofu, 1978; Murayama, 1982). Several others have emphasized the necessity to introduce a variable $\tau$ (e.g. Akasofu, 1981; Vasyliunas, 1987; Gonzalez et al., 1989; Feldstein et al., 1990; Prigancová and Feldstein, 1992).

On the observation basis that the decay time is much shorter during the early recovery of very intense storms, as identified by large negative $D_{s t}$, it has been proposed that $\tau$ depends on $D_{s t}$. In this way, Feldstein et al. (1990) have introduced two values of 10 and $11.5 \mathrm{~h}$ for the decay time parameter according to intervals of $D_{s t}$ during the recovery phase. Gonzalez et al. (1989) have used three $\tau$ values of $4,0.5$, and $0.25 \mathrm{~h}$ for $D_{s t} \geq-50 \mathrm{nT}$, $-50>D_{s t} \geq-120 \mathrm{nT}$, and $D_{s t}<-120 \mathrm{nT}$, respectively. After that, Gonzalez et al. (1993) modified and improved the previous model to introduce very intense storms extending the values of $\tau$ to five, that is, $4.0,2.0,1.0,0.5$, and $0.25 \mathrm{~h}$ for $D_{s t} \geq-50 \mathrm{nT},-50>D_{s t} \geq-100 \mathrm{nT},-100>D_{s t} \geq-200 \mathrm{nT}$, $-200>D_{s t} \geq-300 \mathrm{nT}$, and $D_{s t}<-300 \mathrm{nT}$, respectively. So, typical values of several hours, during the beginning of the main phase of the storm, can arrive to values lower than $1 \mathrm{~h}$ during the main phase peak of very intense storms.

However, it has been reported (e.g. Mendes Jr., 1992) that the decay time $\tau$, considering $D_{s t}$ intervals, results on discontinuities in the relation between the ring current dissipation and the coupling function.

In order to avoid the reported discontinuities in the balance equation, some attempts fitting data parameterized in terms of $D_{s t}$ have been done to provide a continuous functional form (e.g. Valdivia et al., 1996; Monreal MacMahon and Gonzalez, 1997).

Other authors have suggested a control decay by a function determined by the solar wind-magnetosphere coupling. Akasofu (1981) have observed a decay time $\tau$ of about $20 \mathrm{~h}$ for the parameter $\epsilon<5 \times 10^{18} \mathrm{ergs}$ and $\tau \sim 1 \mathrm{~h}$ for $\epsilon>5 \times 10^{18} \mathrm{ergs}$. Pudovkin et al. (1988) found a functional dependence between $\tau$ and a relation proportional to $v B_{S}$. O'Brien and McPherron (2000a) have also proposed that the ring current decay time varies with the injection function proportional to $v B_{S}$ but not with $D_{s t}$, emphasizing that the generally observed dependence of the decay parameter on $D_{s t}$ is actually an alias of the coincidence of intense $D_{s t}$ and intense $v B_{S}$. They obtained functional dependencies, of the decay time $\tau$ and the injection term $Q$ on the convection electric field $v B_{S}$, which are consistent with a positive correlation between $\tau$ and $D_{s t}$ peak. In a later work O'Brien and McPherron (2000b) show that their approach (Model 1) performs better than others two models which also provide the time evolution of $D_{s t}$ in terms of solar wind parameters.

A new model for the prediction of $D_{s t}$ on the basis of the solar wind was introduced by Temerin and $\operatorname{Li}(2002,2006)$. In that case the calculated $D_{s t}$ is a sum of several terms including many parameters which results in a more complicated model than some previous ones. Even though they argue that they have far more data than parameters, a common associated criticism is the idea that with enough free parameters one can fit anything.

Other models use arbitrary mathematical expressions to analyze the dynamics of $D_{s t}$ and suggest that the usual firstorder differential equation introduced by Burton et al. (1975) could be replaced by one of second-order (Klimas et al., 1998; Vassiliadis et al., 1999). They concluded that the decay time depends on the presence of the solar wind input and that presumably $v B_{S}$ can change the magnetospheric electric field and modify the ring current decay.

Here, instead of merely fit some data on previous models of $\tau$ or use arbitrary mathematical expressions to analyze the dynamics, we extend our previous work to derive the analytical function for the decay time from characteristic physical properties of a simplified system which considers that the ring current particles are losing energy mainly through a resistive force associated to collisional, viscous-like and other processes.

Physically, the association between the decay time of the ring current particles and the $D_{s t}$ index is given by the DPS theorem, which states that the magnetic field perturbation caused by the ring current particles is directly proportional to their total kinetic energy.

\subsection{The continuous function for the decay time}

During magnetic storm events, a mixture of the fresh ionospheric and solar wind plasmas besides charged particles in the near-earth nightside plasma sheet are injected into the inner magnetosphere taking part of the westward ring current due to their energization and drift in the geomagnetic field.

As stated by the DPS theorem, the perturbation of the magnetic field is directly proportional to the kinetic energy of the ring current particles.

If we consider that the energization of the ring current satisfies the first order differential equation which involves energy storage only in the magnetic field configuration produced by the ring current and energy dissipation in the ring current itself, we can represent this assumption with a simple $L R$ circuit where the emf $V_{0}$, the inductance $L$ and the resistance $R$ are connected in series. 


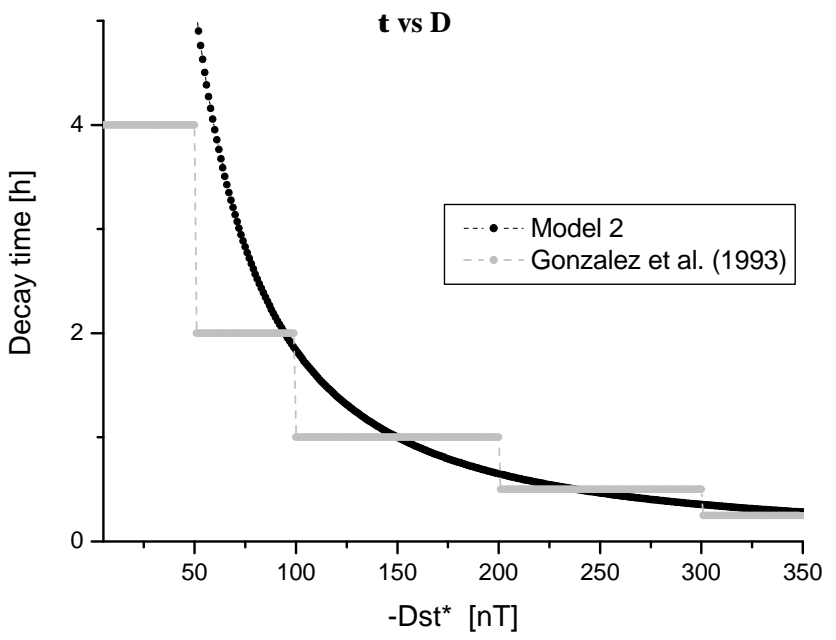

Fig. 1. Two models of the decay time of particles in terms of $D_{s t}$. Comparison between our approach (Model 2) and the refined model of Gonzalez et al. (1993).

There is no ambiguity in considering currents instead of particle flux since the current density is $\mathbf{j}=n q \mathbf{v}$, where $n, q$, and $\mathbf{v}$ are the numeric density, charge, and velocity of particles, respectively.

So, the energy balance

energy input $=$ magnetic storage + ring current dissipation

satisfies the differential equation

$V_{0} i=\frac{d}{d t}\left(\frac{1}{2} L i^{2}\right)+i^{2} R$.

Considering small fluctuations on the magnetic configuration we can assume the autoinductance $L$ of the system as a constant $(d L / d t=0)$. Using the DPS theorem and identifying the kinetic energy of particles $\left(K_{R}\right)$ with the release of particles stored in the magnetic field $\left(\frac{1}{2} L i^{2}\right)$ we arrive to the Burton et al. (1975) model, expressed in Eq. (4).

In this way, the usual balance equation of Burton et al. (1975), when derived from the simple LR-circuit, did not consider temporal variability in the autoinductance. Consequently, we can assume that the variability of the decay time $\tau=L / 2 R$ only depends on the resistance $R$ of the system, that is, on viscous-like, collisional or any other-related resistive process.

Then, for a resistive force depending on the velocity of particles in the ring current, $v$ (note that, as stated before, the electrical drift current $i$ is proportional to the velocity $v$ of charged particles), we can develop the resistive force $F_{r}$ in Taylor's series as follows:

$F_{r}=F_{0}+\left(\frac{\partial F}{\partial v}\right)_{0} v+\frac{1}{2 !}\left(\frac{\partial^{2} F}{\partial v^{2}}\right)_{0} v^{2}+\ldots \ldots$

The speed of particles $v_{R}$ in the preexisting ring current is enhanced by the injection of particles with speed $v_{i}$, so, the net current speed in the ring current is $v=v_{R}+v_{i}$. When the injection of particles stops, the velocity of particles in the ring current attains an extreme value and then begins to decrease returning back to previous values. This means that the resistive force in the ring current never reaches a zero value.

The extreme value is satisfied when $\left(\frac{\partial F}{\partial v}\right)_{0}=0$ and $\left(\frac{\partial^{2} F}{\partial v^{2}}\right)_{0}=\kappa<0$. In a first approach, neglecting the higher order terms, the resistive force results proportional to $v^{2}$, that is

$F_{r}=\kappa v^{2}$.

This is the case when the velocity of particles is greater than the thermal speed (e.g. Molina, 2000; Murray et al., 2004).

As the associated work in the ring current is given by $d W_{r}=\mathbf{F}_{\mathbf{r}} \cdot \mathbf{d l}$, where $d l=v d t$, then, the average work done by the resistive forces during a characteristic decay time $\tau$ is

$<W_{r}>\approx \kappa v^{3} \tau$

So, the decay time is inversely proportional to the cubic power of the speed of particles,

$\tau=\gamma v^{-3}$

But, from the DPS relation the absolute value of the $D_{s t}$ index is proportional to the kinetic energy of particles, that is $D \propto K_{R}$, and as the kinetic energy is proportional to the square of the speed of particles, $K_{R} \propto v^{2}$, we can write for the ring current decay time

$\tau=(\alpha / D)^{3 / 2}$

where $\alpha$ (which is proportional to $\gamma^{2 / 3}$ ) is an adjustable parameter.

Note, from Eqs. (9) and (10), that $\gamma$ is a parameter proportional to $\left\langle W_{r}\right\rangle$. The average work in the ring current is driven by the storm intensity which is characterized by the $-D_{s t}$ peak $D_{0}$, so, the $\alpha$ parameter should depends on $D_{0}$. An early analysis of this parameter during the recovery phase of storms gives a linear relationship between $\alpha$ and $D_{0}$ (Monreal MacMahon et al., 2002). However, the study of this relationship is out of the scope of the present work.

A fit of Eq. (11) with the refined work of Gonzalez et al. (1993) is shown in Fig. 1, where the value of the adjustable parameter $\alpha$ was chosen in order to get a decay time of $1 \mathrm{~h}$ when $D_{s t}^{*}=-150 \mathrm{nT}$.

The model is tested in the next section predicting $D_{s t}$ for a couple of magnetic storms using the chosen constant value for $\alpha$.

\subsubsection{Prediction of $D_{s t}$}

In the previous section we have developed our simple model from an analytical point of view. At this time, it is important to test the behavior of our model predicting $D_{s t}$ for specific observed storm events. 
13 April 1981

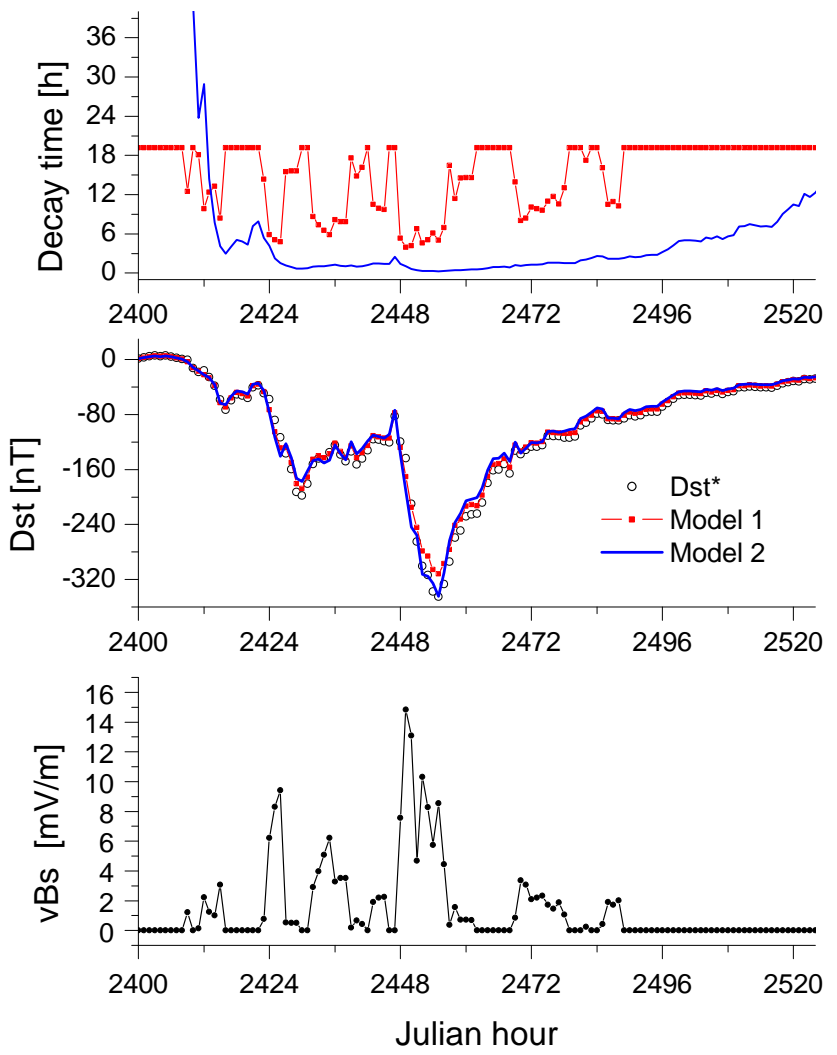

Fig. 2. Event: 11-15 April 1981. The top panel shows time variations of the decay time of ring current particles for models 1 (solid squares) and 2 (continuous line), between 2400 and 2524 Julian Hours, on April of 1981. The middle panel shows a comparison between the $D_{s t}$-forecast of both models and the calculated pressure corrected $D_{s t}$ values (open circles). The bottom panel shows the electric field characterizing the injection.

A $1 \mathrm{~h}$-forecast for different models of the decay time $\tau$ can be done using the discrete version of the Burton et al. equation, that is

$D_{s t}^{*}(t+\Delta t)=D_{s t}^{*}(t)+\left[Q(t)-\frac{D_{s t}^{*}(t)}{\tau}\right] \Delta t$

We use the model of O'Brien and McPherron (2000a) as a good reference (Model 1) to compare with our model (Model 2) because between the models providing the time evolution of $D_{s t}$ in terms of $v B_{S}$ their approach perform best (O'Brien and McPherron, 2002b). For a comparison with models of the decay time based on interplanetary parameters, it is necessary to choose specific sample storms between those we found a good solar wind coverage.

For brevity we have chosen two storms, a complex storm event that occurred on 11-15 April, between 2400 and 2524 Julian hours, of 1981 and the very intense storm that occurred on 1-6 March, between 1416 and 1560 Julian hours, of 1982.

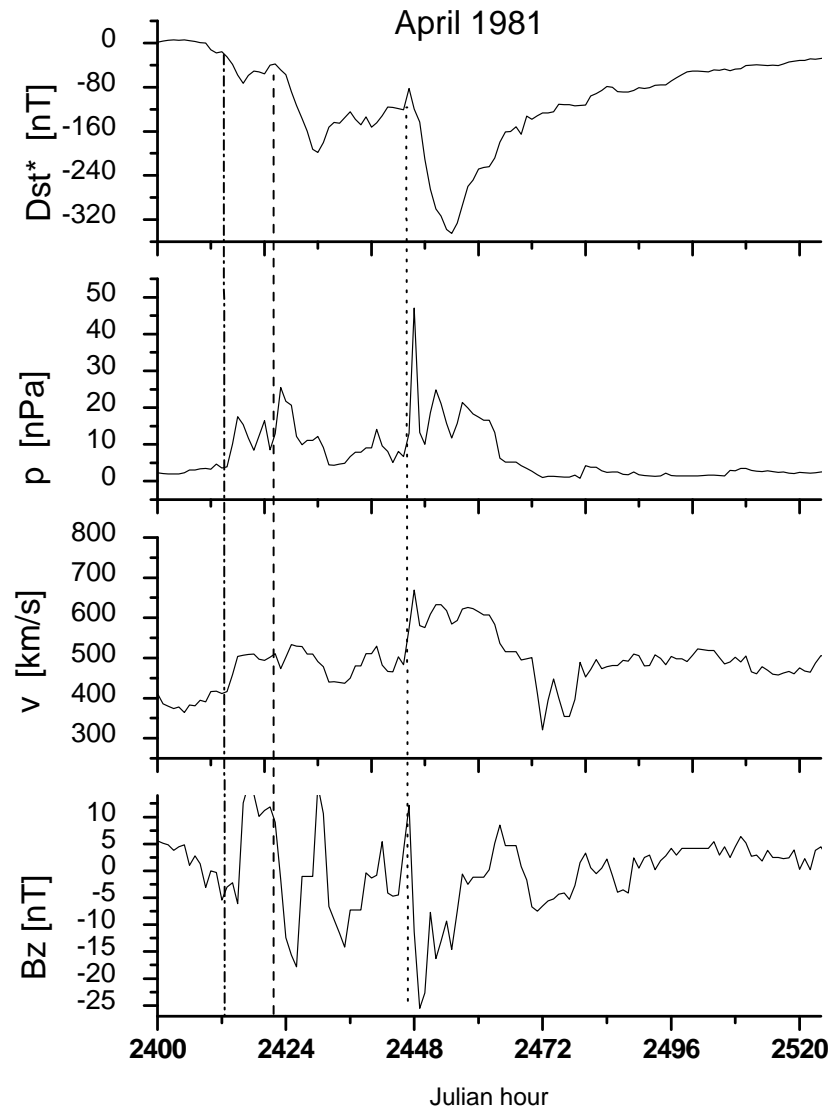

Fig. 3. The top panel shows time variability of the pressure corrected $D_{s t}$ behavior during the event of 11-15 April 1981. The second panel shows time variations of the calculated dynamic pressure for the same event. The third panel shows the time variations of the solar wind velocity. The bottom panel shows the time variability of the IMF- $B_{z}$ component.

Figures 2 and 4 show at the top panel, the behavior of the ring current decay time for model 1 (solid squares) and model 2 (continuous line), at the middle panel a 1-h $D_{s t}$ forecast comparison between both models and the calculated pressure corrected $D_{s t}$ values (open circles), and at the bottom panel the electric field characterizing the energy injection.

Figures 3 and 5 show at the top panel, the time variability of the computed pressure corrected $D_{s t}$ index. The second, third and bottom panels show the time variations of the calculated dynamic pressure, the solar wind velocity and the IMF- $B_{z}$ component, respectively.

It is clear from the top panels of Figs. 2 and 4 that low (high) values of $-D_{s t}$ are related to long (short) decay time of particles. Long decay time values are characteristics of a quiet $D_{s t}$ behavior previous to the storm commencement and both models fit well the observed values. An asymptotic decay time value of around twenty hours (the maximum value in Model 1) is enough and adequate for low $D_{s t}$. 


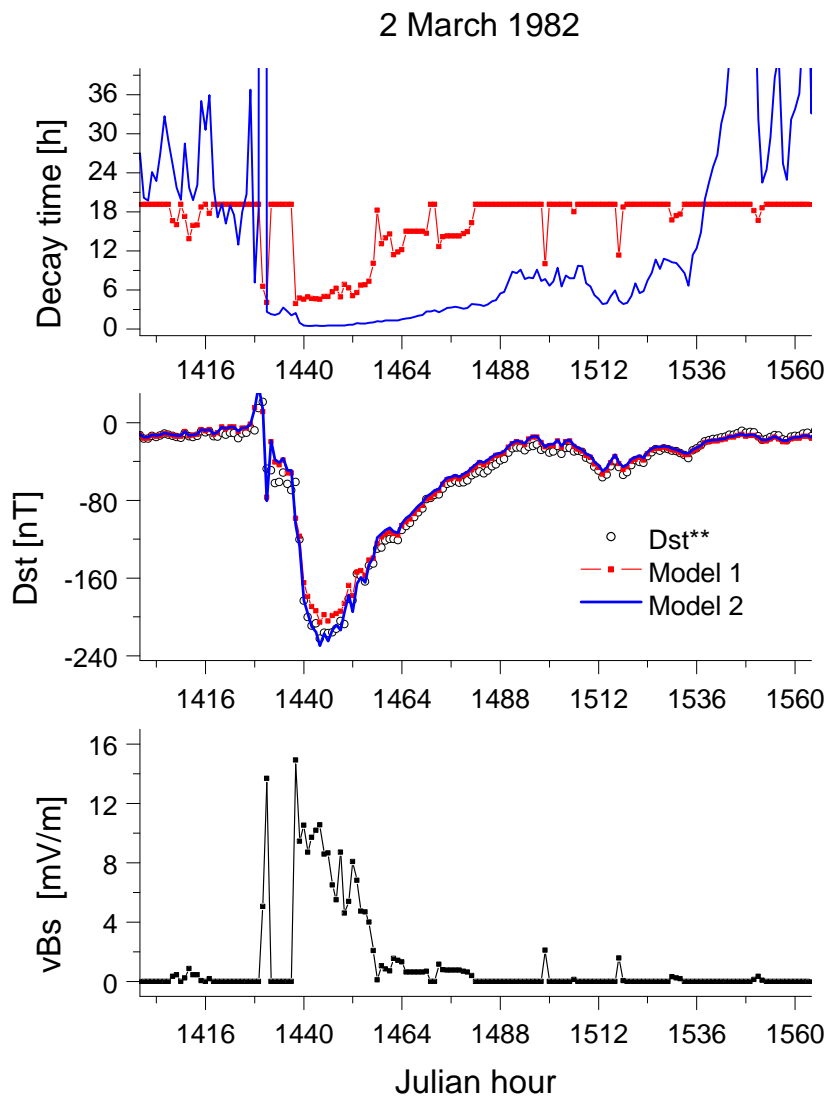

Fig. 4. Event: 1-6 March 1982. Same as that in Fig. 2 except for the period, between 1416 and 1560 Julian Hours on March of 1982.

During stormtime, where the lifetime of particles decreases, our decay time model (continuous line) get shorter values than model 1 (solid squares) and looks like a well behaved smooth function of time.

Figures 2 and 3 show the complex event occurred on April 1981, 2400-2524 Julian hours, which includes a sequence of three types of storms, from moderate to super intense. Prior to the beginning of the whole three-step event, $D_{s t}$ presents a quiet behavior oscillating around zero values for a couple of days. During the occurrence of the first two, a moderate and an intense storm, both models follow quite well (with subtle difference between them) the standard $D_{s t}$ variability (open circles). More significant differences appear between both models during the development of the main and early recovery phase of the last event, a super intense storm $\left(D_{s t}\right.$ peak $<-240 \mathrm{nT}$ ) initiated by a huge amount of energy injection $\left(v B_{s}\right.$ peak $\left.\sim 15 \mathrm{mV} / \mathrm{m}\right)$ which coincides with an interplanetary shock (dotted line in Fig. 3) characterized by sudden changes in solar wind speed $(\Delta v \sim 200 \mathrm{~km} / \mathrm{s}$ in $1 \mathrm{~h})$, high dynamic pressure, and IMF- $B_{z}$ changing in $\sim 40 \mathrm{nT}$, from $\sim 12 \mathrm{nT}$ to $\sim-26 \mathrm{nT}$, in less than $2 \mathrm{~h}$ (Fig. 3). Injection persists (remains over $E_{c}=4.9 \mathrm{mV} / \mathrm{m}$ ) for about eight hours, during the main phase, while solar wind speed and pressure

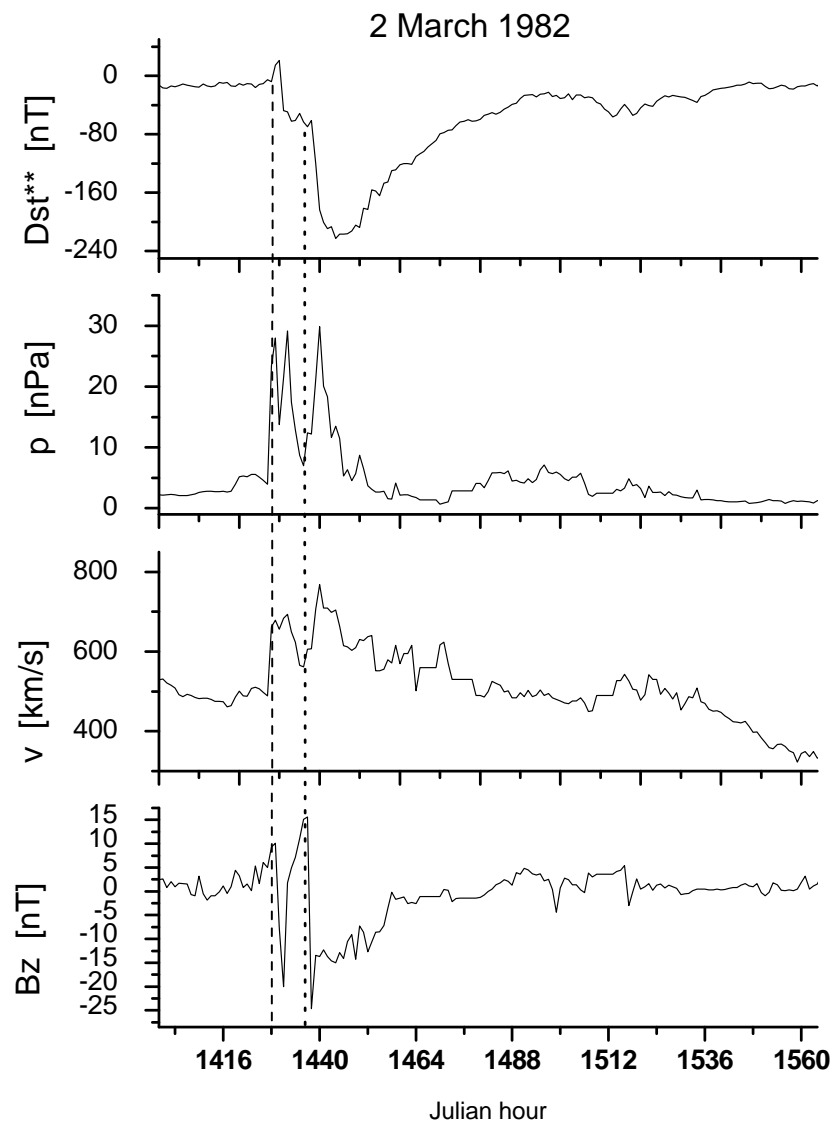

Fig. 5. Same as that in Fig. 3 except for the event of 1-6 March 1982. From top to bottom, the behavior of the pressure corrected $D_{s t}$ index and the interplanetary parameters as dynamic pressure, solar wind speed and the electric field $v B_{S}$.

oscillates. The main phase of this super storm begins with high values of $-D_{s t}$ (around $90 \mathrm{nT}$ ), that is, before the previous intense storm had sufficiently recovered. This probably prevented model 1 from reaching the $D_{s t}$ peak. However, the next recovery of the whole event is clear and both models fit quite well the standard $D_{s t}$.

Figures 4 and 5 show the storm occurred on 1-6 March 1982, 1416-1560 Julian hours (chosen previously by O'Brien and McPherron (2000a) to test their model). The beginning of this large event coincides also with the incidence of an interplanetary shock (dashed line in Fig. 5) associated to abrupt enhancements in dynamic pressure, solar wind speed and interplanetary magnetic field disturbing the quiet $D_{s t}$ behavior. After the storm sudden commencement the main phase develops in two steps. The rapid and large changes of the IMF- $B_{z}$ component, from northward $(\sim 10 \mathrm{nT})$ to southward $(\sim-20 \mathrm{nT})$, initiates the storm main phase injecting energy for a short period through the penetration of a sudden electric field ( $v B_{s}$ peak $\sim 15 \mathrm{mV} / \mathrm{m}$ ). A second step is developed due to a new and more persistent intrusion of IMF- $B_{z}$ changing in $1 \mathrm{~h}$ from $\sim 16 \mathrm{nT}$ to $\sim-24 \mathrm{nT}$ 
(dotted line in Fig. 5). While the injection persists is accompanied by similar but less abrupt enhancements in pressure and solar wind speed. The $D_{s t}$ recovery develops after the injection recedes and all the parameters return back to quiter values. The 1-h forecast of both models (middle panel of Fig. 4) fit quite well the standard $D_{s t}$ behavior during the whole event. Some differences appear again around the $D_{s t}$ peak values.

Even though the models sometimes fail to reach some peak values, especially when an intense storm event develops after a not fully recovered previous storm, the performance of both models is quite good (correlation coefficients $\sim 0.99$ and standard deviations of $\sim 8 \mathrm{nT}$ ). However, by all measures done apparently our simple approach perform best during large storms around peak $D_{s t}$ values.

We assume that the decay time of particles in the ring current depends on $D_{s t}$. Physically, this association is given by the DPS theorem, which states that the magnetic field perturbation caused by the ring current particles is directly proportional to their total kinetic energy. The $D_{s t}$ index is obtained from the horizontal component of the geomagnetic field responding more directly to the internal dynamic of the inner magnetosphere and the ring current decay time should depends not only to changing convection electric fields imposed by solar wind interactions but to internal dynamics of the inner magnetosphere system. The energy injection is primarily driven by the solar wind which enhances the electric field convection related to the generation of cross-tail currents and consequently the flux currents originating in the magnetotail. Part of the energy is redistributed in the inner magnetosphere through different particle fluxes associated to diverse drifts feeding the ring current. The global current flux, involving also plasmas of ionospheric origin, is associated to drift currents and magnetization currents which attains a maximum value during the storm peak identified with the largest absolute value of $D_{s t}$. At this time the resistive forces depending on the velocity of particles intruding the ring current also attains a maximun and consequently the decay time of particles get its lower value. After that, the recovery phase proceeds and the decay time increases as a function of $D$, the absolute value of the $D_{s t}$ index.

\section{Conclusions}

The decay time of particles in the ring current was developed as a continuous function of the absolute value of the pressure corrected $D_{s t}$ index proposed previously. This function was analytically obtained just considering losses by viscous-like and other related processes represented by a resistive force depending on the speed of the particles conforming the ring current. Physically, the association between the decay time of the ring current particles and the $D_{s t}$ index is given by the DPS theorem, which states that the magnetic field perturba- tion caused by the ring current particles is directly proportional to their total kinetic energy.

A comparison with observations of our model and a previous empirical model was done during intense magnetic storms through a forecast analysis using a discrete version of the Burton et al equation. The analysis shows that each of the studied data events is better described introducing our proposed functional form for the decay time parameter in the balance equation. However, our functional form depends also on an adjustable parameter $\alpha$ which was obtained fitting our decay time model to the previous approach of Gonzalez et al. (1993). As it is apparent in Fig. 1, the chosen fit is better correlated for more intense storms. Probably an even better correlation with observation, during storm events, should be obtained considering the dependence of the $\alpha$ parameter on the storm intensity as discussed in Sect. 3. A further analysis on this relationship is out of the scope of the present paper and will be matter of a future work.

Acknowledgements. We would like to thank the NSSDC for making the OMNI dataset available. This work was partially supported by FONDECYT grant 1991020.

Topical Editor I. A. Daglis thanks two anonymous referees for their help in evaluating this paper.

\section{References}

Akasofu, S.-I.: Energy coupling between the solar wind and the magnetosphere, Space Sci. Res., 28, 121-190, 1981.

Akasofu, S.-I.: Some critical issues on magnetospheric substorms, Planet. Space Sci., 34, 563-560, 1986.

Akasofu, S.-I. and Chapman, S.: Solar Terrestrial Physics, Clarendon, Oxford, England, 1972.

Burton, R. K., McPherron, R. L., and Russell, C. T.: An empirical relationship between interplanetary conditions and $D_{s t}$, J. Geophys. Res., 80, 4204-4214, 1975.

Daglis, I. A., Thorne, R. M., Baumjohann, W., and Orsini, S.: The terrestrial ring current: Origin, formation, evolution and decay, Rev. Geophys., 37, 407-438, 1999.

Daglis, I. A., Kozyra, J. U., Kamide, Y., Vassiliadis, D., Sharma, A. S., Liemohn, M. W., Gonzalez, W. D., and Tsurutani, B. T.: Intense space storms: Critical issues and open disputes, J. Geophys. Res., 108(A5), 1208, doi:10.1029/2002JA009722, 2003.

Dessler, A. J. and Parker, E. N.: Hydromagnetic theory of geomagnetic storms, J. Geophys. Res., 64, 2239-2252, 1959.

Ebihara, Y. and Ejiri, M.: Simulation study on fundamental properties of the storm-time ring current, J. Geophys. Res., 105, 15 843-15 859, 2000.

Feldstein, Y. I., Grafe, A., Pisarskij, V., Yu, V., Prigancová, A., and Surnaruk, P. V.: Magnetic field of the magnetospheric ring current and its dynamics during magnetic storms, J. Atmos. Terr. Phys., 52, 1185-1191, 1990.

Frank, L. A.: On the extraterrestrial ring current during geomagnetic storm, J. Geophys. Res., 72, 3753-3767, 1967.

Gonzalez, W. D., Tsurutani, B. T., Gonzalez, A. L. C., Smith, E. J., Tang, F., and Akasofu, S.-I.: Solar wind-magnetosphere coupling during intense magnetic storms (1978-1979), J. Geophys. Res., 94, 8835-8851, 1989. 
Gonzalez, W. D.: Ring current evolution during intense magnetic storm/substorm relationship, Storm/Substorm relationship Workshop, Breckenridge, Colorado, 1993.

Gonzalez, W. D., Joselyn, J. A., Kamide, Y., Kroehl, H. W., Rostoker, G., Tsurutani, B. T., and Vasyliunas, V. W.: What is a geomagnetic storm?, J. Geophys. Res., 99, 5771-5792, 1994.

Klimas, A. J., Vassiliadis, D., and Baker, D. N.: $D_{s t}$ index prediction using data-derived analogues of the magnetospheric dynamics, J. Geophys. Res., 103, 20 435-20 447, 1998.

Langel, R. A. and Estes, R. H.: Large-scale, near-field magnetic fields from external sources and the corresponding induced internal field, J. Geophys. Res., 90, 2487-2494, 1985.

Maltsev, Y. P.: Points of controversy in the study of magnetic storms, Space Sci. Rev., 110, 227-267, 2004.

Mendes Jr., O.: A origem interplanetaria e o desenvolvimento da fase principal das tempestades geomagneticas moderadas (19781979), PhD thesis, INPE-5445-TDI/491, Sao Jose dos Campos, Sao Paulo, Brazil, 1992.

Molina, M. I.: Body motion in a resistive medium at temperature T, Rev. Mex. Fis., 48(2), 132-134, 2000.

Monreal MacMahon, R. and Gonzalez, W. D.: Energetics during the main phase of geomagnetic storms, J. Geophys. Res., 102, 14 199-14 207, 1997.

Monreal MacMahon, R., Llop, C., and Miranda, R.: Ring current decay during northward turnings of the interplanetary magnetic field, Geophys. Res. Abstr., 4, EGS02-A-00577, EGS XXVII General Assembly, Nice, France, 2002.

Murayama, T.: Coupling function between solar wind parameter and geomagnetic indices, Rev. Geophys. Space Phys., 20, 623629, 1982.

Murray, N., Weingartner, J. C., and Capobianco, C.: On the flux of extrasolar dust in Earth-s atmosphere, Astrophys. J, 600, 804827, 2004.

O'Brien, T. P. and McPherron, R. L.: An empirical phase space analysis of ring current dynamics: Solar wind control of injection and decay, J. Geophys. Res., 105, 7707-7719, 2000.

O'Brien, T. P. and McPherron, R. L.: Forecasting the ring current index $D_{s t}$ in real time, J. Atmos. Solar Terr. Phys., 62, 12951299, 2000.
Perrault, P. and Akasofu, S.-I.: A study of geomagnetic storm, Geophys. J. Roy. Astrom. Soc., 54, 547-573, 1978.

Prigancová, A. and Feldstein, Ya. I.: Magnetospheric storm dynamics in terms of energy output rate, Planet. Space Sci., 40, 581588, 1992.

Pudovkin, M. I., Grafe, A., Zaitseva, S. A., Sisova, L. Z., and Usmanov, A. V.: Calculating the Dst-variation field on the basis of solar wind parameters, Gerland Beitr. Geophysik, Leipzig, 97, 525-533, 1998.

Sckopke, N.: A general relation between the energy of trapped particles and the disturbance field near the Earth, J. Geophys. Res., 71, 3125-3130, 1966.

Stern, V. M.: Energetics of the magnetosphere, Space Sci. Rev., 39, 193-213, 1984.

Sun, W. and Akasofu, S.-I.: On the formation of the storm-time ring current belt, J. Geophys. Res., 105, 5411-5418, 2000.

Temerin, M. and Li, X.: A new model for the prediction of $D_{s t}$ on the basis of the solar wind, J. Geophys. Res., 107(A12), 1472, doi:10.1029/ 2001JA007532, 2002.

Temerin, M. and Li, X.: $D_{\text {st }}$ model for 1995-2002, J. Geophys. Res., 111(A04), 221, doi:10.1029/ 2005JA011257, 2006.

Valdivia, J. A., Sharma, A. S., and Papadopoulos, K.: Prediction of magnetic storms by nonlinear models, Geophys. Res. Lett., 23, 2899-2902, 1996.

Vassiliadis D., Klimas, A. J., Valdivia, J. A., and Baker, D. N.: The $D_{s t}$ geomagnetic response as a function of storm main phase and amplitude and the solar wind electric field, J. Geophys. Res., 104, 24 957-24 976, 1999.

Vasyliunas, V. M.: A method for evaluating the total magnetospheric energy output independently of the epsilon parameter, Geophys. Res. Lett., 14, 1183-1186, 1987.

Williams, D. J.: Ring current composition and sources: An update, Planet. Space Sci., 37, 1195-1203, 1981.

Zhang, J., Liemohn, M. W., De Zeeuw, D. L., Borovsky, J. E., Ridley, A. J., Toth, G., Sazykin, S., Thomsen, M. F., Kozyra, J. U., Gombosi, T. I., and Wolf, R. A.: Understanding storm-time ring current development through data-model comparisons of a moderate storm, J. Geophys. Res., 112(A04), 208, doi:10.1029/2006JA011846, 2007. 